\title{
A modified Mediterranean diet score is associated with a lower risk of incident metabolic syndrome over 25 years among young adults: the CARDIA (Coronary Artery Risk Development in Young Adults) study
}

\author{
Lyn M. Steffen ${ }^{1 *}$, Linda Van Horn ${ }^{2}$, Martha L. Daviglus ${ }^{3}$, Xia Zhou ${ }^{1}$, Jared P. Reis ${ }^{4}$, Catherine M. Loria ${ }^{4}$, \\ David R. Jacobs ${ }^{1}$ and Kiyah J. Duffey ${ }^{5}$ \\ ${ }^{1}$ Division of Epidemiology and Community Health, University of Minnesota School of Public Health, \\ 1300 South Second Street, Suite 300, Minneapolis, MN 55454, USA \\ ${ }^{2}$ Northwestern University Feinberg School of Medicine, Chicago, IL, USA \\ ${ }^{3}$ University of Illinois at Chicago, College of Medicine, Chicago, IL, USA \\ ${ }^{4}$ National Heart, Lung, and Blood Institute, National Institutes of Health, Bethesda, MD, USA \\ ${ }^{5}$ Department of Human Nutrition, Foods and Exercise, Virginia Tech, Blacksburg, VA, USA
}

(Submitted 15 March 2014 - Final revision received 24 July 2014 - Accepted 29 July 2014 - First published online 19 September 2014)

\begin{abstract}
The Mediterranean diet has been reported to be inversely associated with incident metabolic syndrome (MetSyn) among older adults; however, this association has not been studied in young African American and white adults. The objective of the present study was to evaluate the association of a modified Mediterranean diet (mMedDiet) score with the 25-year incidence of the MetSyn in 4713 African American and white adults enrolled in the Coronary Artery Risk Development in Young Adults (CARDIA) study. A diet history questionnaire was used to assess dietary intake at baseline, year 7 and year 20 and a mMedDiet score was created. Cardiovascular risk factors were measured at multiple examinations over 25 years. The MetSyn was defined according to the National Cholesterol Education Program Adult Treatment Panel III (ATP III) criteria. Cox proportional-hazards regression analysis was use to evaluate associations for incident MetSyn across the mMedDiet score categories adjusting for demographic characteristics, lifestyle factors and BMI. Higher mMedDiet scores represented adherence to a dietary pattern rich in fruit, vegetables, whole grains, nuts and fish, but poor in red and processed meat and snack foods. The incidence of MetSyn components (abdominal obesity, elevated TAG concentrations and low HDL-cholesterol concentrations) was lower in those with higher mMedDiet scores than in those with lower scores. Furthermore, the incidence of the MetSyn was lower across the five mMedDiet score categories; the hazard ratios and $95 \%$ CI from category 1 to category 5 were 1.0; $0.94(0.76,1.15) ; 0.84(0.68,1.04) ; 0.73(0.58,0.92)$; and $0.72(0.54,0.96)$, respectively $\left(P_{\text {trend }}=0.005\right)$. These findings suggest that the risk of developing the MetSyn is lower when consuming a diet rich in fruit, vegetables, whole grains, nuts and fish.
\end{abstract}

Key words: Metabolic syndrome: Mediterranean dietary pattern: Prospective studies: African Americans

Findings from randomised clinical trials have demonstrated a lower risk of incident and recurrent CVD and their risk factors to be associated with a healthy dietary pattern, including the Mediterranean dietary pattern ${ }^{(1,2)}$. The Mediterranean dietary pattern is a broadly studied dietary pattern, featuring high intake of olive oil, olives, legumes, whole grains, vegetables, and fruit, moderate intake of fish, low-to-moderate intake of dairy products (primarily as cheese and yogurt), moderate intake of wine with meals, and low intake of red meat and processed meat ${ }^{(3)}$

Previous studies have demonstrated inverse associations between the Mediterranean dietary pattern and mortality ${ }^{(4,5)}$ and risk of several chronic conditions ${ }^{(6)}$, including the metabolic syndrome (MetSyn), a cluster of several CVD risk factors. A recent meta-analysis of fifty studies has reported that the Mediterranean diet is associated with a lower risk of the MetSyn $^{(7)}$; however, there was significant heterogeneity in this meta-analysis and the authors concluded that more prospective studies are required to investigate this association ${ }^{(7)}$. Of the previously published prospective studies reporting an inverse association between the Mediterranean diet score and incident MetSyn, three ${ }^{(8-10)}$ were conducted in a Spanish population and in middle-aged US adults. Few studies examining the associations between dietary patterns and the

Abbreviations: CARDIA, Coronary Artery Risk Development in Young Adults; mMedDiet, modified Mediterranean diet; MetSyn, metabolic syndrome. 
MetSyn have used an 'a priori' Mediterranean diet score adapted to the US diet $^{(9)}$, especially in a racially diverse young adult population with 25 years of follow-up.

The purpose of the present study was to determine the longitudinal association of a modified Mediterranean diet (mMedDiet) score with the risk of developing the MetSyn among young black and white adults enrolled in the Coronary Artery Risk Development in Young Adults (CARDIA) study. We hypothesised that the mMedDiet score will be inversely associated with the risk of incident MetSyn over 25 years of follow-up.

\section{Methods \\ Study population}

The CARDIA study is a multi-centre population-based prospective study of CVD risk factor evolution in black and white men and women. Details of the CARDIA study design and participants have been reported previously ${ }^{(11)}$. Briefly, 5115 participants aged 18-30 years were recruited at baseline (1985-6) from Chicago, IL; Minneapolis, MN; Birmingham, AL; and Oakland, CA. A total of seven clinical examinations were conducted at years 0 (baseline), 2, 5, 7, 10, 15, 20 and 25, with follow-up response rates of 91, 86, $81,79,72$ and $72 \%$, respectively ${ }^{(12)}$. Informed consent was obtained from all participants during each examination, and the study was approved by institutional review committees at each site.

Participants were excluded from the present analyses for the following reasons: extreme energy intake at baseline, year 7 and year $20(<3347 \mathrm{~kJ} / \mathrm{d} \quad(<800 \mathrm{kcal} / \mathrm{d})$ and $>33472 \mathrm{~kJ} / \mathrm{d}(>8000 \mathrm{kcal} / \mathrm{d})$ for men and $<2510 \mathrm{~kJ} / \mathrm{d}$ ( $<600 \mathrm{kcal} / \mathrm{d})$ and $>25104 \mathrm{~kJ} / \mathrm{d}(>6000 \mathrm{kcal} / \mathrm{d})$ for women; $n$ 88); pregnancy during the year 0 examination ( $n$ 7); prevalent MetSyn at baseline (defined below, $n$ 110); lack of fasting blood sample at baseline ( $n$ 147). Measurements taken during pregnancy at years 7, 20 and 25 were treated as missing ( $n$ 65). Thus, the sample size for the analyses was 4713 (1030 black men, 1349 black women, 1110 white men and 1224 white women).

\section{Data collection}

Clinical measurements. The participants were asked to fast for at least $8 \mathrm{~h}$ and to avoid smoking and heavy physical activity $2 \mathrm{~h}$ before the examination. Blood was drawn using a standard protocol by venepuncture. Plasma lipid concentrations were determined at the Northwest Lipid Research Clinic Laboratory at the University of Washington (Seattle, WA, USA). In particular, LDL-containing lipoproteins were precipitated with dextran sulphate/magnesium chloride before determining HDL concentrations ${ }^{(13)}$. TAG concentrations were enzymatically measured, using a blank-corrected method $^{(14)}$. Fasting glucose concentrations were quantified using the hexokinase method and standard RIA ${ }^{(15)}$. During each examination, three seated blood pressure measurements were obtained from the right arm using a Hawksley random-zero sphygmomanometer (WA Baum Company) following a $5 \mathrm{~min}$ rest period. The average of the second and third measurements was used in the analyses.

Waist circumference was measured to the nearest $0.5 \mathrm{~cm}$ with a tape in duplicate around the minimal abdominal girth. Body weight was measured to the nearest $0 \cdot 2 \mathrm{~kg}$ with a calibrated balance beam scale. Height was measured with a vertical ruler to the nearest $0.5 \mathrm{~cm}$. BMI was computed as weight in $\mathrm{kg}$ divided by height in $\mathrm{m}^{2}$.

The MetSyn is defined as having at least three of the following five criteria according to the National Cholesterol Education Program Adult Treatment Panel III (ATP III) guidelines ${ }^{(16)}$ : waist circumference $>88 \mathrm{~cm}$ for women or $>102 \mathrm{~cm}$ for men; elevated systolic or diastolic blood pressure $\geq 130$ or $\geq 85 \mathrm{mmHg}$, respectively, or use of antihypertensive medication; elevated fasting TAG concentrations $\geq 1.69 \mathrm{mmol} / 1$ ( $\geq 150 \mathrm{mg} / \mathrm{dl}$ ); fasting glucose concentrations $\geq 6 \cdot 11 \mathrm{mmol} / 1$ ( $\geq 110 \mathrm{mg} / \mathrm{dl}$ ) or having diabetes; low HDL-cholesterol concentrations $(<1.29 \mathrm{mmol} / \mathrm{l} ;<50 \mathrm{mg} / \mathrm{dl})$.

Assessment of dietary intake. During examinations at years 0, 7 and 20, dietary intake was assessed using an interviewer-administered diet history questionnaire specifically developed for the CARDIA study ${ }^{(17)}$. The participants were asked to self-report dietary intake for the past $28 \mathrm{~d}$, including the frequency of food consumption, amount of food consumed, food preparation methods, and type of fat used in food preparation or at the table. The validity and reliability of this diet history questionnaire were evaluated and described in detail ${ }^{(18)}$. Reported foods and beverages were grouped into food groups according to the Nutrient Data Software for Research (NDSR; University of Minnesota, Minneapolis, MN, USA) food grouping scheme ${ }^{(19)}$. Daily intake of each food or beverage group was calculated as the sum of the number of servings consumed per day.

The Mediterranean diet pattern score according to Trichopoulou et al. ${ }^{(4)}$ was modified (mMedDiet) and includes twenty-two food and beverage groups plus a measure of healthy fat intake, i.e., monounsaturated fat + polyunsaturated fat divided by saturated fat (MUFA + PUFA: SFA ratio) with the median as the cut-off. Although Americans do not commonly consume olive oil, they do consume meat, which contributes MUFA to the diet, and vegetable oil, which contributes PUFA to the diet. Therefore, a MUFA + PUFA:SFA ratio was included in the score as an alternative to MUFA intake. Furthermore, present-day food and beverage habits commonly include consumption of coffee, sugar-sweetened beverages and sweet or salty snack foods as reported in both the Mediterranean and US populations ${ }^{(20-23)}$; therefore, the original Mediterranean diet score was modified to include the majority of reported foods and beverages in the diet. Briefly, a value of 0 or 1 was assigned to each of the food and beverage groups and fat ratio. A value of 1 was assigned to individual intakes above the median and 0 to those below the median for foods assumed to be beneficial for health (whole-grain products, fruit, vegetables, fruit + vegetable juice, legumes, nuts, poultry, fish, eggs, coffee and tea, and the MUFA + PUFA:SFA ratio). A value of 1 was assigned to individual intakes below the median and 0 to those above 
the median for foods presumed to be harmful to health (red and processed meat, dairy products, fried vegetables, refined grains, sauces, snack foods, sugar-sweetened beverages and diet beverages). For alcohol, 1 was assigned to men who consumed between 10 and $50 \mathrm{~g} / \mathrm{d}$ and to women who consumed between 5 and $25 \mathrm{~g} / \mathrm{d}$. Thus, the total mMedDiet score ranged from 0 (less healthy dietary pattern) to 19 (more healthy dietary pattern).

Other covariates. Data on demographic characteristics (age, sex, race, field centre and education at baseline) and lifestyle behaviours (physical activity and cigarette smoking) were obtained via standardised questionnaires across CARDIA examination visits. A validated CARDIA physical activity history questionnaire was administered ${ }^{(24)}$, while vitamin supplement use was queried as a separate question in the CARDIA diet history questionnaire ${ }^{(17)}$.

\section{Statistical analyses}

SAS version 9.2 software (SAS Institute) was used for all statistical analyses. Baseline characteristics are reported as means and standard deviations for continuous variables and as frequency (\%) for categorical variables. The cumulative incidence of MetSyn components over 25 years was estimated. The first occurrence of the MetSyn at any of the years 2, 5, 7, $10,15,20$ and 25 is defined as the 25-year incidence of the MetSyn.

The mMedDiet score was divided into five categories. The average number of food servings consumed from each food group was computed within categories of the mMedDiet score using linear regression, adjusting for sex, age, race, education, field centre and energy intake. Time-dependent Cox proportional-hazards regression analysis was used to evaluate associations for the 25-year incidence of the MetSyn, as well as for each MetSyn component, across the mMedDiet score categories and to assess whether these associations were modified by race or sex. Time at risk was defined as until first diagnosis or date of the last follow-up examination. Hazard ratios were computed for category 2 through category 5 of the mMedDiet score compared with category 1 (lowest intake) as the referent group, including interaction terms for race and sex. The cumulative average of dietary intakes, which increases the precision of dietary exposure, was used to create food groups and patterns ${ }^{(25)}$. The time-varying dietary exposures were modelled as (1) between baseline (year 0) and year 7 , dietary exposure was based on dietary intake as measured at year 0; (2) year 7 to year 20, dietary exposure was based on the average of dietary intakes as measured at years 0 and 7; and (3) year 20 to year 25, dietary exposure was based on dietary intake as measured at year 20. A total of four models were developed: model 1, adjusted for energy intake and demographic characteristics (age, race, sex, education and field centre); model 2, adjusted for all variables in model 1 plus current smoking status, physical activity and vitamin supplement use at baseline (as dietary intake may be related to other behaviours); model 3, adjusted for all variables in model 2 plus baseline BMI; model 4, adjusted for all variables in model 3 plus waist circumference at baseline (to determine whether the association between the MetSyn and mMedDiet score was independent of baseline adiposity). A linear trend across the mMedDiet score categories was tested with contrast statements using orthogonal polynomial coefficients. The proportional-hazards assumption was tested and found to be satisfied ${ }^{(26)}$.

\section{Results}

The average number of food servings consumed daily from each food group across the five mMedDiet score categories is summarised in Table 1. Generally, more servings of whole grains, fruit, vegetables, nuts, fish and seafood were consumed by those with higher mMedDiet scores, while fewer servings of these foods were consumed by those with lower scores. Fewer servings of red and processed meat, dairy products, refined grains, snack foods, candy, sugar-sweetened beverages and diet beverages were consumed by those with higher mMedDiet scores than by those with the lowest scores. The proportion of men and women reporting moderate alcohol intake was 51.2 and $48.8 \%$, respectively. Nutrient intake was supportive of dietary intake: fibre, vitamin $\mathrm{C}$ and $\mathrm{Mg}$ intakes were greater, while saturated fat intake was lowest in those with the highest mMedDiet scores than in those with the lowest scores (Table 2).

Baseline characteristics are given in Table 3. Compared with those in the lowest mMedDiet score category, a greater proportion of participants in the highest mMedDiet score category were older, white, more highly educated and more physically active. Furthermore, participants with higher diet scores had lower waist circumference and higher HDL-cholesterol concentrations. The other CVD risk factors were similar across the mMedDiet score categories.

The 25-year cumulative incidence of MetSyn components (abdominal obesity, high TAG concentrations and low HDLcholesterol concentrations) decreased with higher mMedDiet scores (except for elevated glucose concentrations, all $P_{\text {trend }} \leq 0.02$ ) (Table 4 ). Over $59 \%$ of the participants assigned to category 1 of the mMedDiet score were abdominally obese compared with $42 \%$ who reported eating a healthier diet (category 5). Generally, there were fewer participants with adverse MetSyn components in the higher mMedDiet score categories than in the lowest category.

Furthermore, the incidence of the MetSyn was lower with increasing mMedDiet score. Over 25 years of follow-up, hazard ratios and 95\% CI for individuals with mMedDiet scores across categories $1-5$ were 1.0; $0.94(0.76,1.15) ; 0.84$ $(0.68,1.04) ; 0.73(0.58,0.92)$; and $0.72(0.54,0.96)$, respectively, adjusted for age, sex, race, education, field centre, energy intake, smoking status, physical activity and vitamin supplement use (Table 5; model 2, $\left(P_{\text {trend }}=0.005\right)$ ). Additional adjustment for BMI and waist circumference at baseline did not influence the observed associations (models 3 and $\left.4 ; P_{\text {trend }}=0.004\right)$. The proportional-hazards assumption was met for the models $(P>0.05)$. No effect modification by sex or race was observed for the association between the mMedDiet score and incident MetSyn $(P>0 \cdot 05)$. 
Table 1. Average number of daily servings ${ }^{*}$ consumed from each food group across the modified Mediterranean diet (mMedDiet) score categories: CARDIA (Coronary Artery Risk Development in Young Adults) study, $n 4713$

\begin{tabular}{|c|c|c|c|c|c|c|}
\hline \multirow{2}{*}{$\begin{array}{l}\text { Food groups } \\
\text { Category ... }\end{array}$} & \multicolumn{5}{|c|}{ Baseline mMedDiet score } & \multirow[b]{2}{*}{$P$} \\
\hline & $1(n 514)$ & $2(n 1400)$ & $3(n 1843)$ & $4(n 605)$ & $5(n 351)$ & \\
\hline mMedDiet score & $1-8$ & $9-10$ & $11-12$ & $13-14$ & $15-19$ & \\
\hline \multicolumn{7}{|l|}{ Foods (top median scored 1) } \\
\hline Whole grainst & 1.06 & 1.30 & 1.58 & 1.81 & 1.94 & $<0.001$ \\
\hline Fruit & 1.03 & 1.42 & 1.87 & $2 \cdot 27$ & $2 \cdot 68$ & $<0.001$ \\
\hline Juice & $1 \cdot 10$ & $1 \cdot 12$ & $1 \cdot 18$ & $1 \cdot 32$ & 1.24 & 0.07 \\
\hline Vegetables & $2 \cdot 68$ & 3.54 & 4.03 & 5.08 & $5 \cdot 80$ & $<0.001$ \\
\hline Legumes & $0 \cdot 13$ & 0.20 & 0.28 & 0.28 & 0.35 & $<0.001$ \\
\hline Nuts & 0.54 & 0.87 & $1 \cdot 14$ & 1.53 & 2.00 & $<0.001$ \\
\hline Fish and seafood & 0.54 & 0.88 & $1 \cdot 10$ & 1.38 & 1.56 & $<0.001$ \\
\hline Poultry & 1.03 & $1 \cdot 28$ & 1.50 & 1.69 & 1.85 & $<0.001$ \\
\hline Eggs & 0.43 & 0.57 & 0.62 & 0.73 & 0.69 & $<0.001$ \\
\hline Coffee & $1 \cdot 31$ & 1.31 & 1.38 & 1.59 & 1.58 & 0.02 \\
\hline Tea & 0.31 & 0.51 & 0.64 & 0.79 & 1.02 & $<0.001$ \\
\hline \multicolumn{7}{|l|}{ Foods (top median scored 0 ) } \\
\hline Dairy products & $2 \cdot 88$ & $2 \cdot 72$ & $2 \cdot 35$ & 2.09 & 1.74 & $<0.001$ \\
\hline Fried vegetables & 0.31 & 0.23 & $0 \cdot 18$ & 0.13 & 0.08 & $<0.001$ \\
\hline Sauces & $1 \cdot 16$ & $1 \cdot 12$ & 0.97 & 0.92 & 0.70 & $<0.001$ \\
\hline Refined grains $\ddagger$ & 3.53 & $3 \cdot 11$ & 2.55 & $2 \cdot 14$ & 1.42 & $<0.001$ \\
\hline Red/processed meat§ & $3 \cdot 36$ & $3 \cdot 32$ & $2 \cdot 80$ & 2.43 & 2.07 & $<0.001$ \\
\hline Snack foods $\|$ & 0.98 & 0.97 & 0.90 & 0.80 & 0.66 & $<0.001$ \\
\hline Candy & 0.43 & 0.38 & 0.27 & 0.22 & 0.15 & $<0.001$ \\
\hline Sugar group & 1.96 & 1.90 & 1.89 & $1 \cdot 71$ & 1.42 & 0.05 \\
\hline Sugar-sweetened beverages & 1.09 & 0.92 & 0.73 & 0.61 & 0.66 & $<0.001$ \\
\hline Diet beverages & 1.52 & $1 \cdot 17$ & 0.83 & 0.62 & 0.38 & $<0.001$ \\
\hline \multicolumn{7}{|l|}{ Others } \\
\hline Moderate alcohol intake $(\%)^{\star *}$ & $10 \cdot 8$ & $9 \cdot 25$ & $12 \cdot 0$ & $10 \cdot 8$ & $12 \cdot 4$ & 0.07 \\
\hline
\end{tabular}

${ }^{*}$ Adjusted for age, sex, race, education, field centre and energy intake.

† Whole-grain foods without snack foods.

†Refined-grain foods without snack foods.

$\S$ Meat includes red and processed meats, liver and other organ meats.

\|S Snack foods include cake, cookies, pie, pastry, crackers, granola bars, snack chips and popcorn.

I Sugar group includes sugar, honey, syrup, jam and jelly, and sweet sauces.

${ }^{\star \star}$ Moderate alcohol intake for men is $10-50 \mathrm{~g} / \mathrm{d}$ and for women is $5-25 \mathrm{~g} / \mathrm{d}=$ top median scored 1.

\section{Discussion}

In this cohort of young adults, the 25-year incidence of the MetSyn was inversely associated with the adherence to a dietary pattern similar to the Mediterranean dietary pattern characterised by high intake of fruit, vegetables, nuts, fish and seafood, and whole grains, moderate intake of alcohol, and low intake of red and processed meat, dairy products and snack foods. Although the hallmark of the Mediterranean diet is olive oil, we included a MUFA + PUFA:SFA ratio in the mMedDiet score as Americans do not consume olive oil at amounts consumed by the Mediterranean population. Study participants with higher mMedDiet scores were older,

Table 2. Daily nutrient intake* across the modified Mediterranean diet (mMedDiet) score categories at baseline: CARDIA (Coronary Artery Risk Development in Young Adults) study, $n 4713$

\begin{tabular}{|c|c|c|c|c|c|c|}
\hline \multirow{2}{*}{$\begin{array}{l}\text { Nutrient intake } \\
\text { Category... }\end{array}$} & \multicolumn{5}{|c|}{ Baseline mMedDiet score } & \multirow[b]{2}{*}{$P$} \\
\hline & $1(n 514)$ & $2(n 1400)$ & $3(n 1843)$ & $4(n 605)$ & $5(n 351)$ & \\
\hline mMedDiet score & $1-8$ & $9-10$ & $11-12$ & $13-14$ & $15-19$ & \\
\hline Energy & & & & & & 0.005 \\
\hline $\mathrm{kJ}$ & 12652 & 11857 & 12113 & 11489 & 11837 & \\
\hline kcal & 3024 & 2834 & 2895 & 2746 & 2829 & \\
\hline Fibre $(g / d)$ & $17 \cdot 1$ & $19 \cdot 6$ & $22 \cdot 0$ & $24 \cdot 2$ & $26 \cdot 3$ & $<0.001$ \\
\hline Vitamin C (mg/d) & $126 \cdot 9$ & $159 \cdot 5$ & $170 \cdot 7$ & $187 \cdot 9$ & $189 \cdot 9$ & $<0.001$ \\
\hline Vitamin D (mcg/d) & $5 \cdot 36$ & $5 \cdot 77$ & 5.57 & $5 \cdot 79$ & $5 \cdot 91$ & 0.12 \\
\hline $\mathrm{Ca}(\mathrm{mg} / \mathrm{d})$ & $1015 \cdot 9$ & 1025.9 & $1000 \cdot 9$ & $1000 \cdot 2$ & $960 \cdot 0$ & 0.10 \\
\hline $\mathrm{Mg}(\mathrm{mg} / \mathrm{d})$ & $302 \cdot 3$ & $333 \cdot 2$ & $359 \cdot 2$ & $390 \cdot 6$ & $419 \cdot 2$ & $<0.001$ \\
\hline Total fat $(\mathrm{g} / \mathrm{d})$ & $100 \cdot 9$ & $100 \cdot 1$ & $97 \cdot 7$ & 93.6 & 88.5 & $<0.001$ \\
\hline $\operatorname{MUFA}(\mathrm{g} / \mathrm{d})$ & $36 \cdot 5$ & $37 \cdot 2$ & $36 \cdot 9$ & $36 \cdot 1$ & $36 \cdot 5$ & 0.75 \\
\hline PUFA (g/d) & $22 \cdot 2$ & $22 \cdot 1$ & 22.9 & $22 \cdot 0$ & 20.9 & 0.37 \\
\hline $\operatorname{SFA}(g / d)$ & $34 \cdot 3$ & $33 \cdot 1$ & $30 \cdot 3$ & $28 \cdot 2$ & 24.4 & $<0.001$ \\
\hline MUFA + PUFA:SFA & 1.74 & 1.83 & $2 \cdot 02$ & $2 \cdot 14$ & 2.42 & $<0.001$ \\
\hline
\end{tabular}

${ }^{*}$ Adjusted for age, sex, race, education, field centre and energy intake. 
Table 3. Baseline characteristics* across the modified Mediterranean diet (mMedDiet) score categories among the CARDIA (Coronary Artery Risk Development in Young Adults) study participants, 1985-6, n 4713

\begin{tabular}{|c|c|c|c|c|c|c|}
\hline \multirow{2}{*}{$\begin{array}{l}\text { Characteristics } \\
\text { Category... }\end{array}$} & \multicolumn{5}{|c|}{ Baseline mMedDiet score } & \multirow[b]{2}{*}{$P$} \\
\hline & $1(n 514)$ & $2(n 1400)$ & $3(n 1843)$ & $4(n 605)$ & $5(n 351)$ & \\
\hline mMedDiet score & $1-8$ & $9-10$ & $11-12$ & $13-14$ & $15-19$ & \\
\hline Age (years) & $24 \cdot 3$ & 24.4 & $25 \cdot 0$ & $25 \cdot 5$ & $25 \cdot 7$ & $<0.001$ \\
\hline Females (\%) & $62 \cdot 3$ & $55 \cdot 6$ & $51 \cdot 8$ & $51 \cdot 7$ & 54.5 & 0.004 \\
\hline White race (\%) & $46 \cdot 9$ & $45 \cdot 5$ & $49 \cdot 3$ & $54 \cdot 4$ & $62 \cdot 1$ & $<0.001$ \\
\hline Education (above HS) & $61 \cdot 6$ & 45.4 & $59 \cdot 1$ & $69 \cdot 7$ & 67.5 & $<0.001$ \\
\hline Current smoking status (\%) & $29 \cdot 2$ & $29 \cdot 1$ & $31 \cdot 6$ & $27 \cdot 9$ & $24 \cdot 6$ & 0.11 \\
\hline Physical activity score & 361.0 & $401 \cdot 7$ & 431.6 & 447.4 & 463.2 & $<0.001$ \\
\hline $\mathrm{BMI}\left(\mathrm{kg} / \mathrm{m}^{2}\right)$ & $24 \cdot 3$ & $24 \cdot 2$ & 24.5 & $24 \cdot 0$ & $24 \cdot 0$ & 0.26 \\
\hline Waist circumference $(\mathrm{cm})$ & $78 \cdot 1$ & $77 \cdot 1$ & 77.5 & $76 \cdot 6$ & $76 \cdot 2$ & 0.005 \\
\hline Systolic BP (mmHg) & $110 \cdot 3$ & $109 \cdot 8$ & $110 \cdot 6$ & $109 \cdot 6$ & 109.4 & 0.14 \\
\hline Diastolic BP (mmHg) & 68.5 & $68 \cdot 1$ & $68 \cdot 7$ & $68 \cdot 3$ & $68 \cdot 1$ & 0.58 \\
\hline $\mathrm{TAG}(\mathrm{mmol} / \mathrm{l})$ & 0.78 & 0.80 & 0.79 & 0.78 & 0.81 & 0.71 \\
\hline HDL-cholesterol (mmol/l) & 1.35 & 1.37 & 1.40 & 1.40 & 1.39 & 0.012 \\
\hline Glucose $(\mathrm{mmol} / \mathrm{l})$ & 4.55 & 4.57 & 4.57 & 4.54 & 4.58 & 0.75 \\
\hline
\end{tabular}

HS, high school; BP, blood pressure.

${ }^{\star}$ Adjusted for age, sex, race, education, field centre and energy intake.

had more years of education and were more physically active than those with lower scores. Although energy intake was similar across the mMedDiet score categories, participants with lower scores consumed over two daily servings of snack foods high in solid fat and/or added sugar, such as sugar-sweetened beverages, candy, cake, cookies, pie, doughnuts, crackers and chips, compared with those with higher mMedDiet scores who consumed about one daily serving of snack foods. These findings suggest that the food choices of study participants who adhered to a dietary pattern similar to a Mediterranean-type diet are consistent with food recommendations of the 2010 US Dietary Guidelines ${ }^{(27)}$.

Although numerous cross-sectional studies have been conducted examining the Mediterranean diet-MetSyn associations, findings have been inconsistent ${ }^{(7)}$. Our findings, based on 25 years of follow-up among Caucasian and African American adults aged 18-30 years at baseline, were consistent with those of three previously conducted prospective studies examining the same associations, but had fewer years of follow-up ${ }^{(8-10)}$. In a study of 2563Spanish men and women, it was found that individuals adhering to a traditional Mediterranean dietary pattern were $80 \%$ less likely to have the MetSyn over 6 years of follow-up compared with those who did not adhere to this dietary pattern ${ }^{(8)}$. However, one limitation of this study was that the components of the MetSyn were self-reported and not measured by trained observers. In a study of 3232 French men and women, three variations of the Mediterranean diet were found to be related to a lower risk of the MetSyn over 6 years of follow-up ${ }^{(10)}$. Among 2730 middle-aged white adult men and women free of diabetes and enrolled in the Framingham Offspring Study, the 7-year incidence of the MetSyn was found to be $21 \%$ lower among those in the highest quintile of a Mediterranean dietary pattern than in those in the lowest quintile ${ }^{(9)}$. Despite differences in study design and methods, including the differing age and race composition of the study populations, dietary intake assessment instrument, data analysis approach and length of follow-up, the associations between the diverse Mediterranean dietary patterns and the MetSyn

Table 4. Incidence of metabolic syndrome components* over 25 years across the modified Mediterranean diet (mMedDiet) score categories: CARDIA (Coronary Artery Risk Development in Young Adults) study, 1985-2010, n 4713

\begin{tabular}{|c|c|c|c|c|c|c|}
\hline \multirow{2}{*}{$\begin{array}{l}\text { Components } \\
\text { Category... }\end{array}$} & \multicolumn{5}{|c|}{ mMedDiet score } & \multirow[b]{2}{*}{$P$} \\
\hline & $1(n 514)$ & $2(n 1400)$ & $3(n 1843)$ & $4(n 605)$ & $5(n 351)$ & \\
\hline mMedDiet score & $1-8$ & $9-10$ & $11-12$ & $13-14$ & $15-19$ & \\
\hline Abdominal obesity (\%)† & $59 \cdot 4$ & $40 \cdot 2$ & $40 \cdot 5$ & $41 \cdot 7$ & $41 \cdot 9$ & $<0.001$ \\
\hline Elevated BP (\%)‡ & $49 \cdot 2$ & $38 \cdot 8$ & $41 \cdot 3$ & $44 \cdot 6$ & $40 \cdot 4$ & $0 \cdot 10$ \\
\hline High TAG (\%)§ & $37 \cdot 3$ & $27 \cdot 9$ & $27 \cdot 8$ & $29 \cdot 9$ & $21 \cdot 6$ & $<0.001$ \\
\hline Low HDL-C (\%)\| & $68 \cdot 4$ & $57 \cdot 5$ & $56 \cdot 4$ & $58 \cdot 0$ & $59 \cdot 3$ & 0.02 \\
\hline Elevated glucose (\%)ף & $21 \cdot 3$ & $15 \cdot 3$ & 14.5 & $14 \cdot 7$ & $19 \cdot 1$ & 0.36 \\
\hline
\end{tabular}

BP, blood pressure; HDL-C, HDL-cholesterol.

*Adjusted for age, sex, race, education, field centre and energy intake.

† Abdominal obesity is defined as waist circumference $>88 \mathrm{~cm}$ for women and $>102 \mathrm{~cm}$ for men

$\ddagger$ Elevated $B P=$ systolic $B P>130 \mathrm{mmHg}$ or diastolic $\mathrm{BP}>85 \mathrm{mmHg}$ or antihypertensive medication use.

$\S$ High TAG $=$ TAG concentrations $\geq 1.69 \mathrm{mmol} / \mathrm{l}(\geq 150 \mathrm{mg} / \mathrm{dl})$.

\| Low HDL-C $=$ HDL-C concentrations $<1.29 \mathrm{mmol} / \mathrm{l}(<50 \mathrm{mg} / \mathrm{dl})$.

१ी Elevated glucose $=$ glucose concentrations $>6.11 \mathrm{mmol} / \mathrm{l}(>110 \mathrm{mg} / \mathrm{dl})$. 
Table 5. Association between a modified Mediterranean diet (mMedDiet) score and risk of developing the metabolic syndrome (MetSyn) over 25 years among young adults enrolled in the CARDIA (Coronary Artery Risk Development in Young Adults) study (Hazard ratios (HR) and $95 \%$ confidence intervals, $n$ 4713)

\begin{tabular}{|c|c|c|c|c|c|c|c|c|c|c|}
\hline \multirow{3}{*}{$\begin{array}{l}\text { Models } \\
\text { Category... }\end{array}$} & \multicolumn{9}{|c|}{ mMedDiet score } & \multirow[b]{3}{*}{$P$} \\
\hline & \multirow[b]{2}{*}{$1(n 514)$} & \multicolumn{2}{|c|}{$2(n 1400)$} & \multicolumn{2}{|c|}{$3(n 1843)$} & \multicolumn{2}{|c|}{$4(n 605)$} & \multicolumn{2}{|c|}{$5(n 351)$} & \\
\hline & & $\mathrm{HR}$ & $95 \% \mathrm{Cl}$ & $\mathrm{HR}$ & $95 \% \mathrm{Cl}$ & $\mathrm{HR}$ & $95 \% \mathrm{Cl}$ & $\mathrm{HR}$ & $95 \% \mathrm{Cl}$ & \\
\hline MedDiet score & $1-8$ & \multicolumn{2}{|r|}{$9-10$} & \multicolumn{2}{|c|}{$11-12$} & \multicolumn{2}{|c|}{$13-14$} & \multicolumn{2}{|c|}{$15-19$} & \\
\hline MetSyn cases $(n)$ & 171 & & 328 & & 06 & & 39 & & 3 & \\
\hline Model $1^{*}$ & $1 \cdot 0$ & 0.91 & $0 \cdot 74,1 \cdot 14$ & 0.77 & $0.63,0.94$ & 0.64 & $0.51,0.81$ & 0.62 & $0.47,0.82$ & $<0.001$ \\
\hline Model $2 \dagger$ & 1.0 & 0.94 & $0.76,1.15$ & 0.84 & $0.68,1.04$ & 0.73 & $0.58,0.92$ & 0.72 & $0.54,0.96$ & 0.005 \\
\hline Model 3‡ & 1.0 & 0.82 & $0.67,1.00$ & 0.76 & $0.62,0.92$ & $0 \cdot 71$ & $0.56,0.91$ & 0.67 & $0.50,0.90$ & 0.004 \\
\hline Model 4§ & 1.0 & 0.82 & $0.67,1.01$ & 0.77 & $0.64,0.94$ & 0.72 & $0.56,0.92$ & 0.67 & $0.49,0.90$ & 0.004 \\
\hline
\end{tabular}

* Model 1 adjusted for age, sex, race, field centre, education and energy intake.

† Model 2 adjusted for all variables in model 1 plus current smoking status, physical activity and vitamin/mineral supplement use.

$\ddagger$ Model 3 adjusted for all variables in model 2 plus BMI.

$\S$ Model 4 adjusted for all variables in model 3 plus waist circumference at baseline.

were consistent between the present study and the other three prospective studies ${ }^{(8-10)}$.

The Mediterranean dietary pattern includes foods and beverages that are rich in a myriad of nutrients, including monounsaturated fat, antioxidants, fibre, $\mathrm{Ca}, \mathrm{Mg}$, and phytooestrogens, and other food components ${ }^{(28)}$. The combined influence of these foods (nutrients and food components) has been shown to confer beneficial effects on the individual components of the MetSyn, including a better lipid profile ${ }^{(29)}$ and lower systolic and diastolic blood pressure ${ }^{(30)}$, adiposity $^{(31)}$ and insulin resistance ${ }^{(32)}$. Evidence from studies of individual food intakes relative to MetSyn components may also explain underlying mechanisms. In addition, inverse associations have been reported between whole-grain foods and glucose concentrations, insulin resistance and incident diabetes $^{(33)}$. Moderate alcohol intake has been reported to be associated with higher HDL-cholesterol concentrations ${ }^{(34)}$. Blood pressure may also be beneficially modulated by intakes of fruit and vegetables that are rich in $\mathrm{K}$, fibre and antioxidants $^{(35)}$, while intakes of plant protein and foods from plant sources are inversely associated with blood pressure ${ }^{(36)}$ and elevated blood pressure ${ }^{(35)}$. Meat intake has been reported to be positively associated with blood pressure and risk of hypertension in several populations ${ }^{(35,37)}$; numerous studies have also shown positive associations between red and processed meat intake and adiposity ${ }^{(38)}$ and type 2 diabetes $^{(39)}$. Finally, fish intake has been reported to be inversely associated with CVD risk factors and the MetSyn ${ }^{(40,41)}$ and its individual components (blood pressure, glucose homeostasis and lipids) ${ }^{(41-43)}$

Olive oil, rich in MUFA and antioxidants, is a characteristic component of the Mediterranean diet and known for its beneficial effects on CVD risk factors, including lipids, blood pressure, and glucose homeostasis, as well as endothelial function, inflammation and oxidative stress ${ }^{(44)}$. However, Americans do not consume large quantities of olives or olive oil, but they do consume other vegetable oils that are high in PUFA, Thus, the MUFA + PUFA:SFA ratio was greater among adults with a higher mMedDiet score. Higher mMedDiet scores were related to higher intake of polyunsaturated fat and lower intake of monounsaturated fat and saturated fat.
Limitations of the present study include reliance on selfreported dietary intake, which may introduce considerable measurement error ${ }^{(45)}$. However, it is likely that dietary intake was ranked correctly. Moreover, dietary intake was assessed by trained and certified interviewers at three visits using the validated CARDIA diet history questionnaire ${ }^{(17,18)}$, ensuring greater precision of dietary intake compared with a single assessment at baseline ${ }^{(25)}$. Finally, residual confounding may still exist even though statistical models were adjusted for numerous potential factors that may confound the association between dietary intake and incident MetSyn.

The present study has several strengths, including the prospective design and study population of African Americans and white men and women aged 18-30 years at baseline, long duration of follow-up, and multiple clinic examinations to characterise the components of the MetSyn and assess incident MetSyn. Finally, the CARDIA diet history questionnaire allowed for the collection of data on specific fat and oil consumption/use in food preparation and added to foods at the table - which was important to calculate the MUFA + PUFA:SFA ratio included in the mMedDiet score

The findings of the present study with 25 years of follow-up suggest that the risk of incident MetSyn in young Caucasian and African American adults is lower when consuming a dietary pattern rich in fruit, vegetables, whole grains, nuts and fish. Promoting a Mediterranean dietary pattern is beneficial to cardiovascular health.

\section{Acknowledgements}

The authors thank the CARDIA study participants for their continued participation in the study.

The CARDIA study was supported by contracts N01HC-48047, N01-HC-48048, N01-HC-48049, N01-HC-48050, N01-HC-95095, and N01-HC-45134 from the National Heart, Lung, and Blood Institute (NHLBI) at the National Institutes of Health. Drs Loria and Reis, who are employed by the NHLBI, contributed to the study design, conduct of the study, interpretation of the findings, and preparation of the manuscript. 
The authors' contributions are as follows: L. M. S. and L. V. H. designed the research; L. M. S., L. V. H. and M. L. D. wrote the first draft of the manuscript; L. M. S. supervised the statistical analyses; X. Z. performed the statistical analyses; L. M. S. had primary responsibility for the final content and is the guarantor of the manuscript, having had full access to the data in the study, and takes responsibility for the integrity of the data and the accuracy of the data analysis. All authors reviewed the manuscript, participated in the interpretation of the study results and approved the manuscript.

None of the authors has any conflicts of interest to declare.

\section{References}

1. Estruch R, Ros E, Salas-Salvadó J, et al. (2013) Primary prevention of cardiovascular disease with a Mediterranean diet. N Engl J Med 368, 1279-1290.

2. Martinez-Gonzalez MA \& Bes-Rastrollo M (2014) Dietary patterns, Mediterranean diet, and cardiovascular disease. Curr Opin Lipidol 25, 20-26.

3. Ministry of Health and Welfare (1999) Dietary guidelines for adults in Greece. Arch Hellen Med 16, 516-524.

4. Trichopoulou A, Costacou T, Bamia C, et al. (2003) Adherence to a Mediterranean diet and survival in a Greek population. New Engl J Med 348, 2599-2608.

5. Sofi F, Cesari F, Abbate R, et al. (2008) Adherence to Mediterranean diet and health status: meta-analysis. BMJ 337, a1344.

6. Martinez-Gonzalez MA, Bes-Rastrollo M, Serra-Majem L, et al. (2009) Mediterranean food pattern and the primary prevention of chronic disease: recent developments. Nutr Rev $\mathbf{6 7}$, S111-S116.

7. Kastorini CM, Milionis HJ, Esposito K, et al. (2011) The effect of Mediterranean diet on metabolic syndrome and its components: a meta-analysis of 50 studies and 534,906 individuals. J Am Coll Cardiol 57, 1299-1313.

8. Tortosa A, Bes-Rastrollo M, Sanchez-Villegas A, et al. (2007) Mediterranean diet inversely associated with the incidence of metabolic syndrome: the SUN prospective cohort. Diabetes Care 30, 2957-2959.

9. Rumawas ME, Meigs JB, Dwyer JT, et al. (2009) Mediterranean-style dietary pattern, reduced risk of metabolic syndrome traits, and incidence in the Framingham Offspring Cohort. Am J Clin Nutr 90, 1608-1614.

10. Kesse-Guyot E, Ahluwalia N, Lassale C, et al. (2013) Adherence to Mediterranean diet reduces the risk of metabolic syndrome: a 6-year prospective study. Nutr Metab Cardiovasc Dis 23, 677-683.

11. Friedman GD, Cutter GR, Donahue RP, et al. (1988) CARDIA: study design, recruitment, and some characteristics of the examined subjects. J Clin Epidemiol 41, 1105-1116.

12. Chamberlain AM, Schreiner PJ, Fornage M, et al. (2009) Ala54Thr polymorphism of the fatty acid binding protein 2 gene and saturated fat intake in relation to lipid levels and insulin resistance: the Coronary Artery Risk Development in Young Adults (CARDIA) study. Metabolism 58, $1222-1228$.

13. Warnick GR, Benderson J \& Albers JJ (1982) Dextran sulfate$\mathrm{Mg}^{2+}$ precipitation procedure for quantitation of highdensity-lipoprotein cholesterol. Clin Chem 28, 1379-1388.

14. Warnick GR (1986) Enzymatic methods for quantification of lipoprotein lipids. Methods Enzymol 129, 101-123.

15. Folsom AR, Jacobs DR, Wagenknecht LE, et al. (1996) Increase in fasting insulin and glucose over seven years with increasing weight and inactivity of young adults. The CARDIA Study. Am J Epidemiol 144, 235-246.

16. Grundy SM, Cleeman JI, Daniels SR, et al. (2005) Diagnosis and management of the metabolic syndrome: an American Heart Association/National Heart, Lung, and Blood Institute Scientific Statement. Circulation 112, 2735-2752.

17. McDonald A, Van Horn L, Slattery M, et al. (1991) The CARDIA dietary history: development, implementation, and evaluation. J Am Diet Assoc 91, 1104-1112.

18. Liu K, Slattery M, Jacobs DR, et al. (1994) A study of the reliability and comparative validity of the CARDIA dietary history. Ethn Dis 4, 15-27.

19. Nutrition Coordinating Center \& University of Minnesota (2005) Nutrition Data System for Research (NDSR), Food and Nutrient Database. http://www.ncc.umn.edu/products/ databasefoodsnutrientsfoodgroups.html (accessed February 2014)

20. Scholer M (2006) Coffee consumption. http://www.worldmapper.org/posters/worldmapper_1038_coffee_consumption_ ver2.pdf (accessed February 2014).

21. Naska A, Orfanos P, Chloptsios Y, et al. (2005) Dietary habits in Greece: The European Prospective Investigation into Cancer and nutrition (the EPIC project). Arch Hellen Med 22, 259-269.

22. Fotiadou E \& Babajimopoulos M (2006) Snack patterns of Greek adults 20-50 years of age. J Food Service 17, $197-204$.

23. Piernas C \& Popkin B (2010) Snacking increased among U.S. adults between 1977 and 2006. J Nutr 140, $325-332$.

24. Jacobs DR Jr, Ainsworth BE, Hartman TJ, et al. (1993) A simultaneous evaluation of 10 commonly used physical activity questionnaires. Med Sci Sports Exerc 25, 81-91.

25. Hu FB, Stampfer MJ, Rimm E, et al. (1999) Dietary fat and coronary heart disease: a comparison of approaches for adjusting for total energy intake and modeling repeated dietary measurements. Am J Epidemiol 149, 531-540.

26. Hosmer DW, Lemeshow S \& May S (2008) Applied Survival Analysis: Regression Modeling of Time to Event Data, 2nd ed. New York: John Wiley \& Sons, Inc.

27. Center for Nutrition Policy and Promotion \& U.S. Department of Agriculture (2011) ChooseMyPlate.gov. http:// www.choosemyplate.gov (accessed November 2013).

28. Kafatos A, Verhagen H, Moschandreas J, et al. (2000) Mediterranean diet of Crete: foods and nutrient content. J Am Diet Assoc 100, 1487-1493.

29. Tzima N, Pitsavos C, Panagiotakos DB, et al. (2007) Mediterranean diet and insulin sensitivity, lipid profile and blood pressure levels, in overweight and obese people; the Attica study. Lipids Health Dis $\mathbf{6}, 22$.

30. Psaltopoulou T, Naska A, Orfanos P, et al. (2004) Olive oil, the Mediterranean diet, and arterial blood pressure: the Greek European Prospective Investigation into Cancer and Nutrition (EPIC) study. Am J Clin Nutr 80, 1012-1018.

31. Babio N, Bullo M \& Salas-Salvado J (2009) Mediterranean diet and metabolic syndrome: the evidence. Public Health Nutr 12, 1607-1617.

32. Salas-Salvadó J, Bullo M, Babio N, et al. (2011) Reduction in the incidence of type 2 diabetes with the Mediterranean Diet: results of the PREDIMED-Reus Nutrition Intervention Randomized trial. Diabetes Care 34, 14-19.

33. McKeown NM, Meigs JB, Liu S, et al. (2002) Whole-grain intake is favorably associated with metabolic risk factors for type 2 diabetes and cardiovascular disease in the Framingham Offspring Study. Am J Clin Nutr 76, 390-398. 
34. de Silva Foster ER, Harper MM, Seidman CE, et al. (2000) Alcohol consumption raises HDL-cholesterol levels by increasing the transport rate of apolipoproteins A-I and A-II. Circulation 102, 2347-2352.

35. Steffen LM, Kroenke CH, Yu X, et al. (2005) Associations of plant foods, dairy products, and meat intakes with $15-\mathrm{y}$ incidence of elevated blood pressure in young black and white adults: The CARDIA Study. Am J Clin Nutr 82, $1169-1177$

36. Elliott P, Stamler J, Dyer AR, et al. (2006) Association between protein intake and blood pressure: the INTERMAP Study. Arch Int Med 166, 79-87.

37. Miura K, Greenland P, Stamler J, et al. (2004) Relation of vegetable, fruit, and meat intake to 7-year blood pressure change in middle-aged men: the Chicago Western Electric Study. Am J Epidemiol 159, 572-580.

38. Wang Y \& Beydoun MA (2009) Meat consumption is associated with obesity and central obesity among US adults. Int J Obes 33, 621-628.

39. Aune D, Ursin G \& Veierød MB (2009) Meat consumption and the risk of type 2 diabetes: a systematic review and meta-analysis of cohort studies. Diabetologia 52, 2277-2287.
40. Baik I, Abbott RD, Curb JD, et al. (2011) Intake of fish and $n$-3 fatty acids and future risk of metabolic syndrome. J Am Diet Assoc 110, 1018-1026.

41. Lara JJ, Economou M, Wallace AM, et al. (2007) Benefits of salmon eating on traditional and novel vascular risk factors in young, non-obese healthy subjects. Atherosclerosis 193, 213-221.

42. Singer P, Berger I, Lück K, et al. (1986) Long-term effect of mackerel diet on blood pressure, serum lipids and thromboxane formation in patients with mild essential hypertension. Atherosclerosis 62, 259-265.

43. Panagiotakos DB, Zeimbekis A, Boutziouka V, et al. (2007) Long-term fish intake is associated with better lipid profile, arterial blood pressure, and blood glucose levels in elderly people from Mediterranean islands (MEDIS epidemiological study). Med Sci Monit 13, CR307-CR312.

44. Covas MI, Konstantinidou V \& Fito M (2009) Olive oil and cardiovascular health. J Cardiovasc Pharmacol 54, 477-482.

45. Neuhouser ML, Tinker L, Shaw PA, et al. (2008) Use of recovery biomarkers to calibrate nutrient consumption selfreports in the Women's Health Initiative. Am J Epidemiol 167, 1247-1259. 